\title{
Intraocular pressure during neurosurgical procedures in context of head position and loss of cerebrospinal fluid
}

\author{
*Patrick Czorlich, MD,', Theresa Krätzig, MD,1 Nikolas Kluge,, Christos Skevas, MD, ${ }^{2}$ \\ Volker Knospe, MD, ${ }^{2}$ Martin Stephan Spitzer, MD, ${ }^{2}$ Marc Dreimann, MD, ${ }^{3}$ \\ Klaus Christian Mende, MD, ${ }^{1}$ Manfred Westphal, MD, ${ }^{1}$ and Sven Oliver Eicker, MD1

\begin{abstract}
Departments of ${ }^{1}$ Neurosurgery, ${ }^{2}$ Ophthalmology, and ${ }^{3}$ Trauma, Hand and Reconstructive Surgery, University Medical Center Hamburg-Eppendorf, Hamburg, Germany
\end{abstract}

\begin{abstract}
OBJECTIVE Perioperative visual loss (POVL) is a rare but serious complication in surgical disciplines, especially in spine surgery. The exact pathophysiology of POVL remains unclear, but elevated intraocular pressure (IOP) is known to be part of it. As POVL is rarely described in patients undergoing intracranial or intradural surgery, the aim of this study was to investigate the course of IOP during neurosurgical procedures with opening of the dura mater and loss of CSF.

METHODS In this prospective, controlled trial, 64 patients fell into one of 4 groups of 16 patients each. Group A included patients undergoing spine surgery in the prone position, group B patients had intracranial procedures in the prone position, and group C patients were treated for intracranial pathologies in a modified lateral position with the head rotated. In groups A-C, the dura was opened during surgery. Group D patients underwent spine surgeries in the prone position with an intact dura. IOP was measured continuously pre-, peri-, and postoperatively.
\end{abstract}

RESULTS In all groups, IOP decreased after induction of anesthesia and increased time dependently after final positioning for the operation. The maximum IOP in group A prior to opening of the dura was $28.6 \pm 6.2 \mathrm{~mm} \mathrm{Hg}$ and decreased to $23.44 \pm 4.9 \mathrm{~mm} \mathrm{Hg}$ directly after dura opening $(p<0.0007)$. This effect lasted for 30 minutes $(23.5 \pm 5.6 \mathrm{~mm}$ $\mathrm{Hg}, \mathrm{p}=0.0028)$; after 60 minutes IOP slowly increased again $(24.5 \pm 6.3 \mathrm{~mm} \mathrm{Hg}, p=0.15)$. In group $B$, the last measured IOP before CSF loss was $28.1 \pm 5.0 \mathrm{~mm} \mathrm{Hg}$ and decreased to $23.5 \pm 6.1 \mathrm{~mm} \mathrm{Hg}(p=0.0039)$ after dura opening. A significant IOP decrease in group B lasted at 30 minutes $(23.6 \pm 6.0 \mathrm{~mm} \mathrm{Hg}, p=0.0039)$ and 60 minutes $(23.7 \pm 6.0$ $\mathrm{mm} \mathrm{Hg}, p=0.0189$ ). In group $C$, only the lower eye showed a decrease in IOP up to 60 minutes after loss of CSF (opening of dura, $p=0.0007 ; 30$ minutes, $p=0.0477 ; 60$ minutes, $p=0.0243$ ). In group $D$ (control group), IOP remained stable throughout the operation after the patient was prone.

CONCLUSIONS This study is the first to demonstrate that opening of the dura with loss of CSF during neurosurgical procedures results in a decrease in IOP. This might explain why POVL predominantly occurs in spinal but rarely in intracranial procedures, offers new insight to the pathophysiology of POVL, and provides the basis for further research and treatment of POVL.

German Clinical Trials Register (DRKS) no.: DRKS00007590 (drks.de)

https://thejns.org/doi/abs/10.3171/2018.3.JNS173098

KEYWORDS intraocular pressure; neurological surgery; prone position; supine position; perioperative visual loss; spine surgery

$\mathrm{T}$ TeMPORARY or permanent perioperative visual loss (POVL) or visual disturbance is an infrequent but serious adverse event primarily reported in patients undergoing spine surgery in the prone position. The rate of POVL ranges between $0.03 \%$ and $0.28 \%$ depending on the surgical procedure. ${ }^{18,32,37}$ Currently, the pathomechanism of POVL is a matter of debate, but 3 causative connec- tions are favored by most studies, namely, ischemic optic neuropathy (ION), central retinal artery occlusion, or cortical blindness. ${ }^{3,18,20}$ ION may occur as either anterior ION, defined as ischemic damage of the optic nerve before it enters the lamina cribrosa sclerae, or posterior ION, defined as an ischemic event of the optic nerve behind the lamina cribrosa sclerae. ${ }^{22}$ ION has been observed to be

ABBREVIATIONS BP = blood pressure; ION = ischemic optic neuropathy; $I O P=$ intraocular pressure; $P O V L=$ perioperative visual loss SUBMITTED December 11, 2017. ACCEPTED March 19, 2018.

* P.C. and T.K. contributed equally to this work. 
the main pathology leading to POVL, with anterior ION being predominantly observed in cardiac surgical cases due to reduced ocular perfusion and posterior ION being described in prone-position spine procedures or laparoscopic surgery in the Trendelenburg position due to reduced venous drainage. ${ }^{11,17,30}$ Besides the aforementioned pathomechanisms, some rare cases of POVL are related to mechanical damage, resulting in an injured cornea with support of the development of ION. ${ }^{23,30}$

The occurrence of ION is closely related to the blood supply of the eye and especially to the blood supply to the optic nerve. ${ }^{38}$ It has been reported that the blood supply depends on the eye's perfusion pressure, flow resistance, intraocular pressure (IOP), rheological factors of the blood, and the presence of autoregulation. ${ }^{12}$ The perfusion pressure within the blood vessels of the optic nerve is the difference between the mean arterial blood pressure (BP) and the IOP. ${ }^{44}$ Many authors have described changes of the IOP depending on the surgical positioning of the patient, with an increase in IOP in the prone or Trendelenburg position and a longer time in these positions due to the duration of surgery. ${ }^{4,6,15,43}$ In addition to prone positioning, some other risk factors, such as obesity, male sex, use of a Wilson frame for spine procedures, blood volume, anemia, and large intraoperative volumes of crystalloids, have been described to be associated with POVL. 14,21,22,24,28 Most of these risk factors also apply to neurosurgical procedures, but POVL has only been reported in single case reports of patients after craniotomy or in patients undergoing ventriculography. ${ }^{35,41}$ Taking into consideration that an elevated IOP might lead to a lower eye perfusion pressure and subsequently to ION resulting in POVL, we hypothesized that the opening of the dura mater and the resulting loss of CSF in patients undergoing craniotomies in the prone position or intradural spine surgery leads to a decrease in the IOP and improves the perfusion pressure of the optic nerve sheath. To confirm the hypothesis of a decrease in the IOP, we evaluated its course in different neurosurgical procedures and compared the findings with those of a control group of patients undergoing spine surgery in the prone position without opening of the dura mater.

\section{Methods}

The protocol of this prospective observational study was registered in the German Clinical Trials Register (registration no. DRKS00007590, drks.de). The study was approved by the local ethics committee of the medical association of the state of Hamburg.

All patients admitted to our institution were screened using inclusion and exclusion criteria to participate in the study. Inclusion criteria were 1) intracranial pathology and intraoperative lateral positioning with the head rotated, prone position, or the necessity of spine surgery with or without loss of CSF in the prone position; 2) written informed consent of the patient; and 3) expected compliance of the patient for completion of the study protocol.

Exclusion criteria were defined as 1) evident or suspected pregnancy; 2) visual acuity of less than 0.05 ; 3) ophthalmological disease, especially any kind of glaucoma or astigmatism with more than \pm 8 diopters; 4) known aller- gic reaction to local anesthesia or mydriatic medication; 5) diabetes mellitus, peripheral vascular diseases, or a BMI $>30 \mathrm{~kg} / \mathrm{m}^{2}$; 6) participation in any other interventional study; 7) any disease that interacted with this study; 8) any kind of neurosurgical emergency intervention with no time for the patient to consider if he/she wanted to participate in this study; and 9) any other aspect that did not allow for participation. Disturbance of eye motility due to pathology in the fossa posterior was not an exclusion criterion.

In accordance with the inclusion and exclusion criteria, all patients were divided into 4 groups as follows: group A, spinal intradural procedures in the prone position with anteroposterior positioning of the head; group $\mathrm{B}$, intracranial procedures with opening of the dura mater in the prone position with anteroposterior positioning of the head, including decompression for Chiari malformation; group $\mathrm{C}$, intracranial procedures in the lateral position with bolstering and rotation of the head; and group D, spine procedures in the prone position with anteroposterior positioning of the head and no loss of CSF. If the dura mater was opened accidentally during surgery in patients allocated to group D, the patient was moved into group A. Usage of a Mayfield skull clamp was mandatory in all patients to perform the intraoperative IOP measurements.

\section{Ophthalmological Examination}

After written informed consent was obtained, all patients were examined by a neuro-ophthalmologist prior to surgery. Ophthalmological examination included bestcorrected visual acuity, anterior chamber evaluation, funduscopy (assessment of the optic nerve head, macula, and peripheral retina), and a pupillary reaction test. Funduscopy was performed after pharmacological mydriasis (Mydriaticum Stulln). Goldmann tonometry was used pre- and postoperatively to measure the IOP with the patient seated. A special disinfected prism was mounted on the tonometer head and then carefully placed against the cornea. A topical anesthetic was used prior to contact with the cornea (Conjucain EDO [oxybuprocaine hydrochloride]). ${ }^{2}$

General anesthesia was induced intravenously with $0.2-0.3 \mathrm{mg} / \mathrm{kg}$ sufentanil and $2-3 \mathrm{mg} / \mathrm{kg}$ propofol or $0.2-$ $0.3 \mathrm{mg} / \mathrm{kg}$ etomidate. Intravenous atracurium $(0.5 \mathrm{mg} / \mathrm{kg})$ or succinylcholine $(1-1.5 \mathrm{mg} / \mathrm{kg}$ ) was used to facilitate tracheal intubation. Anesthesia was maintained with propofol $(5-8 \mathrm{mg} / \mathrm{kg} / \mathrm{hr})$ as total intravenous anesthesia.

After induction of general anesthesia and intubation of the patient, the IOP was measured using a hand-held applanation tonometer (Tono-Pen XL) with an Ocu-Film Tip Cover (Reichert Technologies) with the patient in the supine position and immediately after reaching the final position for the operation. During the procedure, the IOP was measured every 30 minutes and additionally after opening of the dura mater with maximum loss of CSF and again after closure of the dura mater. The final 2 IOP measurements were obtained just before and right after returning the patient to the supine position. In patients in groups A, $\mathrm{B}$, and $\mathrm{D}$, the IOP measurement was performed in a single eye to reduce the risk of cornea irritation (Fig. 1). In patients with their heads rotated (group C), IOP was measured in both eyes, as head rotation was found to cause a difference in the IOP. ${ }^{9}$ 


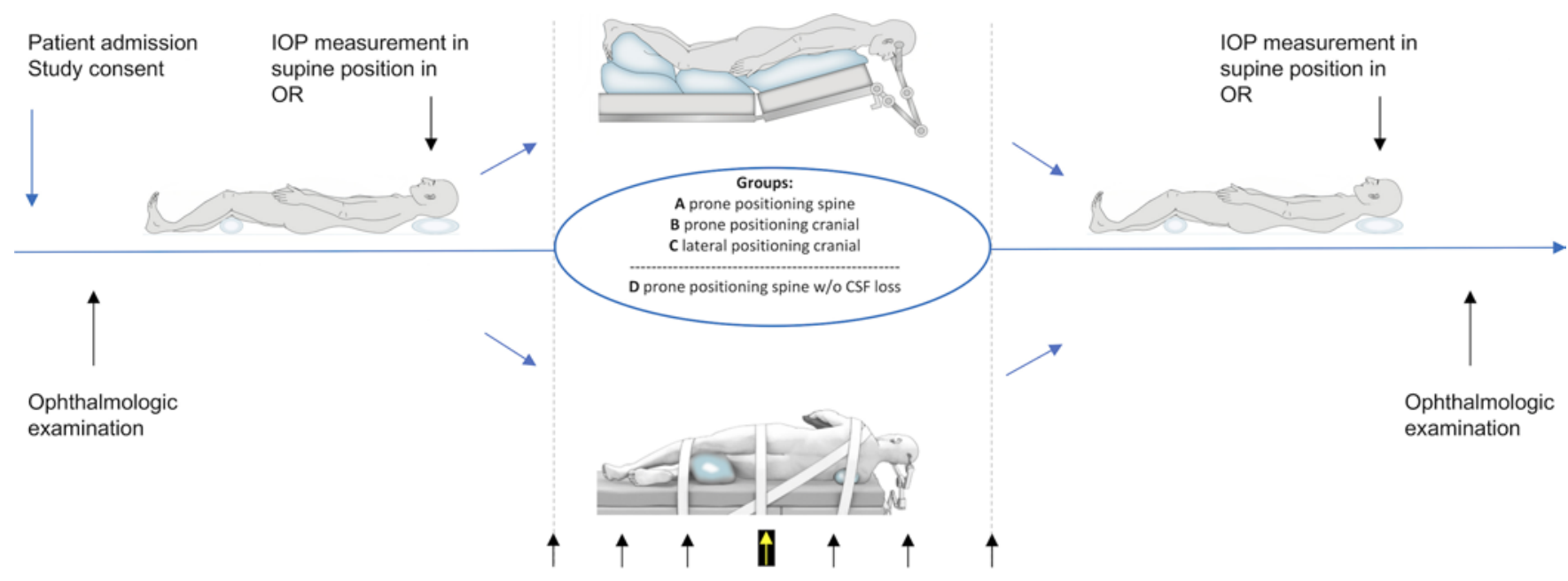

IOP measurement every subsequent $30 \mathrm{~min}$ and at time point of CSF loss

FIG. 1. Flowchart of the study protocol. After obtaining written consent for the study, the first ophthalmological examination, including measurement of the IOP, was performed. Perioperative IOP was measured after the beginning of anesthesia, after final prone or lateral positioning, and subsequently every 30 minutes. An additional measurement was performed after opening the dura mater and CSF loss. Final IOP measurements were obtained at the end of the prone/lateral position and in a supine position followed by a second ophthalmological examination before discharge. $\mathrm{OR}=$ operating room.

During anesthesia, systolic and diastolic BPs were recorded parallel to the IOP measurements. The volume of blood loss and the intraoperative volume of crystalloids were documented at the end of the surgical procedure.

All patients underwent examination by a neuro-ophthalmologist after surgery and prior to discharge as described above to exclude any new pathology of the eyes and to measure the IOP again with the patient seated. When patients reported a visual disturbance or corneal irritation after awakening from anesthesia, a consultation by a neuro-ophthalmologist was provided around the clock to determine the further diagnostic and treatment steps independent from the study protocol.

\section{Statistical Analysis}

In designing the study, a calculation to determine the necessary number of participants was performed. To reach a level of significance of $<0.05$ with an assumed decrease of the IOP of $3 \mathrm{~mm} \mathrm{Hg}$ after opening of the dura mater, an $\alpha$ of 0.05 , and a power of 0.8 for groups $\mathrm{A}-\mathrm{C}$; and an increase of $5 \mathrm{~mm} \mathrm{Hg}$ also with an $\alpha$ of 0.05 and a power of 0.8 for group $\mathrm{D}$, the calculation recommended enrolling 16 patients in each group.

Statistical analysis of the acquired data was performed using GraphPad Prism 7.0 (GraphPad Software), and results were deemed significant at $\mathrm{p}<0.05$. Paired Student t-tests were used for comparing our repeated-measures data. One-way ANOVA was used for the analysis of crystalloid volume and blood loss in combination with Pearson's correlations calculated using IBM SPSS (version 22, IBM Corp.).

\section{Results}

\section{Patient Population}

The recruitment of the calculated cohort of 64 patients was completed between April 2015 and October 2017, consisting of the 4 different treatment groups with 16 patients each; $35.9 \%$ of all patients were male $(12.5 \%$ in group A, $50.0 \%$ in group B, $43.8 \%$ in group C, and $37.5 \%$ in group $\mathrm{D}$ ). The mean patient age was $52.1 \pm 15.1$ years, with no significant age difference in the respective groups (A: $50.19 \pm 13.6$; B: $49.75 \pm 19.5$; C: $55.06 \pm 15.3$; and D: $53.38 \pm 12.4$ years). The mean overall BMI was $23.9 \pm 3.4$ $\mathrm{kg} / \mathrm{m}^{2}$ without significant intergroup differences (A: 24.8 \pm 3.5 ; B: $24.2 \pm 2.6$; C: $24.3 \pm 3.4$; and D: $22.5 \pm 4.0 \mathrm{~kg} /$ $\mathrm{m}^{2}$ ). Diagnosis and localization of the pathologies are presented in detail in Tables 1 and 2.

\section{IOP Measurements}

\section{Group A}

The mean IOP before surgery was $15.6 \pm 2.2 \mathrm{~mm} \mathrm{Hg}$ (range 11-18 mm Hg), decreasing to a mean of $10.5 \pm 3.5$ $\mathrm{mm} \mathrm{Hg}$ (range 6-19 mm Hg, p < 0.0001) after induction of anesthesia and increasing to a mean of $23.0 \pm 5.2 \mathrm{~mm}$ $\mathrm{Hg}$ (range $15-34 \mathrm{~mm} \mathrm{Hg}, \mathrm{p}<0.0001$ ) after prone positioning. During surgery, the mean IOP was $28.6 \pm 6.2 \mathrm{~mm}$ $\mathrm{Hg}$ (range $19-40 \mathrm{~mm} \mathrm{Hg}$ ) at the last measurement before opening of the dura mater. The IOP decreased to a mean of $23.44 \pm 4.9 \mathrm{~mm} \mathrm{Hg}$ (range $12-30 \mathrm{~mm} \mathrm{Hg}, \mathrm{p}<0.0007$ ) after opening of the dura and maximum CSF loss. Significant IOP reduction was preserved at 30 minutes (23.5 $\pm 5.6 \mathrm{~mm} \mathrm{Hg}$, range $9-31 \mathrm{~mm} \mathrm{Hg}, \mathrm{p}=0.0028$ ) but not at 60 minutes after CSF drainage $(24.5 \pm 6.3 \mathrm{~mm} \mathrm{Hg}$, range $17-35 \mathrm{~mm} \mathrm{Hg}, \mathrm{p}=0.15$ ) compared with the last measured values before CSF drainage.

\section{Group B}

In group B, the mean IOP before surgery was $15.4 \pm 2.5$ $\mathrm{mm} \mathrm{Hg}$ (range 11-20 $\mathrm{mm} \mathrm{Hg}$ ) with decrease to a mean of $12.4 \pm 3.5$ mm Hg (range 8-19 mm Hg, p = 0.0052) after 
TABLE 1. Patient diagnoses in all groups

\begin{tabular}{lcccc}
\hline \multicolumn{1}{c}{ Diagnosis } & Group & Group & Group & Group \\
& A & B & C & D \\
\hline Metastasis* $^{*}$ & 1 & 3 & 5 & 2 \\
\hline Glioblastoma WHO grade IV & & 2 & 1 & \\
\hline $\begin{array}{l}\text { Ependymoma WHO grade I or II } \\
\text { Pilocytic astrocytoma WHO } \\
\quad 1\end{array}$ & 1 & 1 & & \\
$\quad$ grade I & & & & \\
\hline $\begin{array}{l}\text { Hemangioblastoma WHO grade I } \\
\text { Meningioma WHO grade I }\end{array}$ & 6 & 1 & 1 & \\
\hline $\begin{array}{l}\text { Neurofibroma/schwannoma } \\
\quad \text { WHO grade I† }\end{array}$ & 2 & & 3 & \\
\hline Trigeminal neuralgia & & & & \\
\hline Cavernoma & 3 & 1 & & \\
\hline Chiari malformation & & 3 & & 1 \\
\hline Degenerative spinal disease & & & & 13 \\
\hline Other & 2 & 2 & 2 & \\
\hline
\end{tabular}

* Lung, breast, rectum, and colon cancers.

† Including vestibular schwannoma.

$\ddagger$ Including vertebral fractures and abscesses.

induction of anesthesia and an increase to a mean of $24.1 \pm$ $3.3 \mathrm{~mm} \mathrm{Hg}$ (range $16-28 \mathrm{~mm} \mathrm{Hg}, \mathrm{p}<0.0001$ ) after prone positioning. The mean IOP at the last measurement before opening of the dura mater was $28.1 \pm 5.0 \mathrm{~mm} \mathrm{Hg}$ (range $19-37 \mathrm{~mm} \mathrm{Hg}$ ), decreasing to a mean of $23.5 \pm 6.1 \mathrm{~mm} \mathrm{Hg}$ (range $10-33 \mathrm{~mm} \mathrm{Hg}, \mathrm{p}=0.0039$ ) after dural opening with loss of CSF. A significant IOP reduction was preserved at 30 minutes $(23.6 \pm 6.0 \mathrm{~mm} \mathrm{Hg}$, range $12-34 \mathrm{~mm} \mathrm{Hg}, \mathrm{p}=$ $0.0039)$ and at 60 minutes $(23.7 \pm 6.0 \mathrm{~mm} \mathrm{Hg}$, range $10-33$ $\mathrm{mm} \mathrm{Hg}, \mathrm{p}=0.0189)$ after CSF drainage.

\section{Group C}

\section{Lower Eye}

Before and after induction of anesthesia, the mean IOPs for the lower eye were $14.6 \pm 2.7 \mathrm{~mm} \mathrm{Hg}$ (range 10-20 mm $\mathrm{Hg}$ ) and $10.7 \pm 6.1 \mathrm{~mm} \mathrm{Hg}$ (range 1-24 mm Hg, $\mathrm{p}=0.05$ ), respectively. It increased to a mean of $18.2 \pm 5.2 \mathrm{~mm} \mathrm{Hg}$ (range $11-30 \mathrm{~mm} \mathrm{Hg}, \mathrm{p}=0.0002$ ) after prone positioning. The maximum mean IOP without CSF loss was $19.8 \pm 5.8$ $\mathrm{mm} \mathrm{Hg}$ (range $12-32 \mathrm{~mm} \mathrm{Hg}$ ), with decrease to a mean of $14.7 \pm 5.5 \mathrm{~mm} \mathrm{Hg}$ (range $7-24 \mathrm{~mm} \mathrm{Hg}, \mathrm{p}=0.0007$ ) after opening of the dura mater. A significant reduction in IOP was preserved at 30 minutes $(16.5 \pm 4.0 \mathrm{~mm} \mathrm{Hg}$, range $7-23 \mathrm{~mm} \mathrm{Hg}, \mathrm{p}=0.0477)$ and at 60 minutes $(17.6 \pm$ $2.5 \mathrm{~mm} \mathrm{Hg}$, range $14-21 \mathrm{~mm} \mathrm{Hg}, \mathrm{p}=0.0243$ ) after CSF drainage compared with the last measured values before CSF drainage.

\section{Upper Eye}

The mean IOP for the upper eye before surgery was 14.9 $\pm 2.4 \mathrm{~mm} \mathrm{Hg}$ (range $10-20 \mathrm{~mm} \mathrm{Hg}$ ). The IOP decreased to a mean of $11.4 \pm 5.4 \mathrm{~mm} \mathrm{Hg}$ (range 4-21 $\mathrm{mm} \mathrm{Hg}, \mathrm{p}=$ 0.012 ) after anesthesia and intubation and increased again to a mean of $14.4 \pm 3.5 \mathrm{~mm} \mathrm{Hg}$ (range 9-21 mm Hg, p = $0.0155)$ after prone positioning.

During surgery, the mean IOP was $14.3 \pm 6.9 \mathrm{~mm} \mathrm{Hg}$ (range 8-27 $\mathrm{mm} \mathrm{Hg}$ ) at the last measurement before open-
TABLE 2. Localization of pathologies in groups $A$ and $D$

\begin{tabular}{lcc}
\hline \multicolumn{1}{c}{ Localization } & Group A & Group D \\
\hline Craniocervical junction & 3 & 1 \\
\hline Cervical spine & 5 & 9 \\
\hline Cervicothoracic junction & 1 & \\
\hline Thoracic spine & 7 & 4 \\
\hline Lumbar spine & & 2 \\
\hline
\end{tabular}

All pathologies in groups $B$ and $C$ were intracranial pathologies. Pathologies that were located within more than 1 vertebral column segment, e.g., from C6 to T2, were defined as cervicothoracic junction and similarly for pathologies that were located within the posterior fossa and the cervical spine as craniocervical junction.

ing of the dura mater. IOP levels decreased to a mean of $12.3 \pm 4.8 \mathrm{~mm} \mathrm{Hg}$ (range $4-17 \mathrm{~mm} \mathrm{Hg}, \mathrm{p}=0.4001$ ) after maximum CSF drainage. No significant IOP reduction was recorded at 30 minutes $(11.7 \pm 4.4 \mathrm{~mm} \mathrm{Hg}$, range 6-21 $\mathrm{mm} \mathrm{Hg}, \mathrm{p}=0.0830)$ or at 60 minutes $(12.0 \pm 4.0 \mathrm{~mm} \mathrm{Hg}$, range 6-16 $\mathrm{mm} \mathrm{Hg}, \mathrm{p}=0.3632$ ) after $\mathrm{CSF}$ drainage compared with the last measured values before CSF drainage.

Comparison of Mean IOP Levels Between the Upper and Lower Eyes

There were no significant differences in IOP between the upper and the lower eyes at anesthesia induction (11.4 vs $10.9 \mathrm{~mm} \mathrm{Hg}, \mathrm{p}=0.81$ ), but the differences were significant after initial lateral positioning (14.4 vs $18.2 \mathrm{~mm} \mathrm{Hg}$, p $=0.0231)$. Differences were also significant at 30 minutes before CSF drainage (15.0 vs $19.8 \mathrm{~mm} \mathrm{Hg}, \mathrm{p}=0.0306$ ). There was no statistically significant difference at the time of dural opening (12.7 vs $14.7 \mathrm{~mm} \mathrm{Hg}, \mathrm{p}=0.4114)$. At 30 minutes (11.8 vs $16.5 \mathrm{~mm} \mathrm{Hg}, \mathrm{p}=0.0061$ ), 60 minutes (12.3 vs $17.6 \mathrm{~mm} \mathrm{Hg}, \mathrm{p}=0.0029)$, and 90 minutes (13.6 vs $18.3 \mathrm{~mm} \mathrm{Hg}, \mathrm{p}=0.0327$ ), however, the differences were statistically significant, whereas after repositioning (15.6 vs $18.4 \mathrm{~mm} \mathrm{Hg}, \mathrm{p}=0.0514)$, there was no significant difference.

\section{Group D}

In group $\mathrm{D}$, the mean IOP before surgery was $16.2 \pm$ $3.3 \mathrm{~mm} \mathrm{Hg}$ (range 9-20 mm Hg) with a decrease to 11.7 $\pm 4.1 \mathrm{~mm} \mathrm{Hg}$ (range 6-23 mm Hg, p = 0.0029) after intubation and an increase to a mean of $23.6 \pm 6.0 \mathrm{~mm} \mathrm{Hg}$ (range $13-35 \mathrm{~mm} \mathrm{Hg}, \mathrm{p}=0.0001$ ) after prone positioning. As a time point before opening of the dura could not be detected in group D, we chose the beginning of surgery as another certain measurement. The mean IOP was $28.6 \pm$ $8.7 \mathrm{~mm} \mathrm{Hg}$ (range $21-45 \mathrm{~mm} \mathrm{Hg}$ ), and there was no significant alteration in IOP during the procedure at $30 \mathrm{~min}-$ utes $(27.14 \pm 5.3 \mathrm{~mm} \mathrm{Hg}, \mathrm{p}=0.4547), 60$ minutes (29.27 $\pm 4.6 \mathrm{~mm} \mathrm{Hg}, \mathrm{p}=0.7837)$, or 90 minutes $(30.6 \pm 4.2 \mathrm{~mm}$ $\mathrm{Hg}, \mathrm{p}=0.6673$ ).

Details of IOP measurement are presented in Figs. 2 and 3 .

\section{Perioperative Blood Pressure}

The mean BP (systolic/diastolic) in group A after induction of anesthesia was $101 \pm 18 / 58 \pm 13 \mathrm{~mm} \mathrm{Hg}$ in group A, $105 \pm 18 / 60 \pm 12 \mathrm{~mm} \mathrm{Hg}$ in group B, $112 \pm 17 / 54$ 


\section{Reduction in IOP Group A}

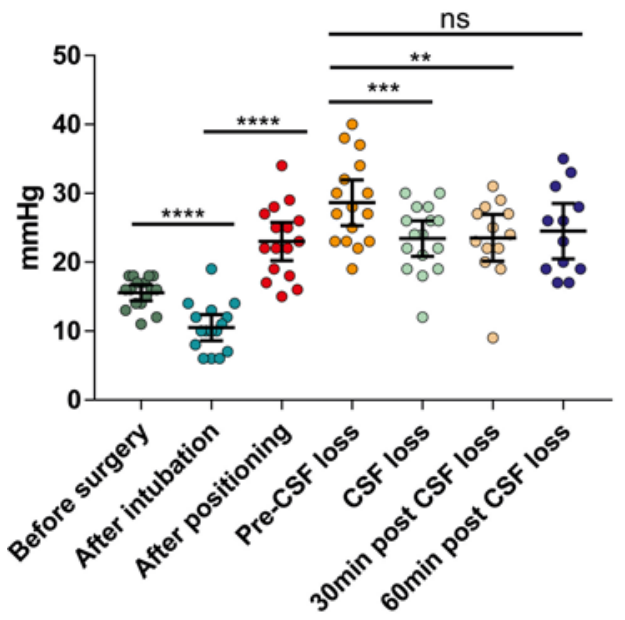

Reduction in IOP Group C LE

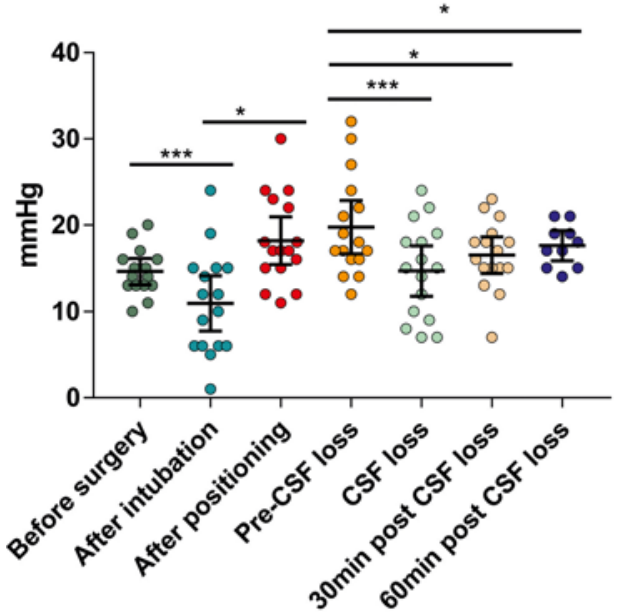

Reduction in IOP Group D

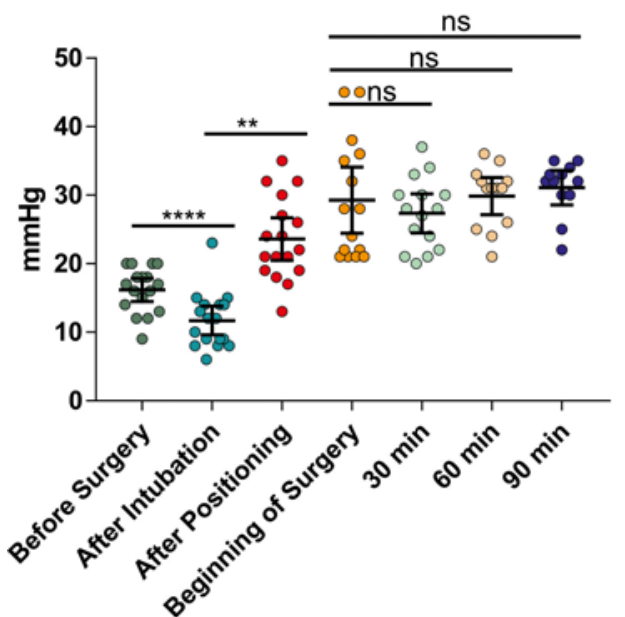

FIG. 2. Reduction of IOP in groups $A, B$, and $D$. Group $C$ is presented in detail, with IOPs demonstrated separately for the upper eye (UE) and lower eye (LE). Distribution of IOPs in each group before surgery, after intubation, after final positioning for surgery, before and after CSF loss, and 30 and 60 minutes after CSF loss. Group D lacks IOP findings for CFS loss as the dura mater was kept intact. ns $=$ not significant. ${ }^{*} p<0.01 ;{ }^{* *} p<0.001 ;{ }^{* * *} p<0.0001 ;{ }^{* * * *} p<0.00001$. 


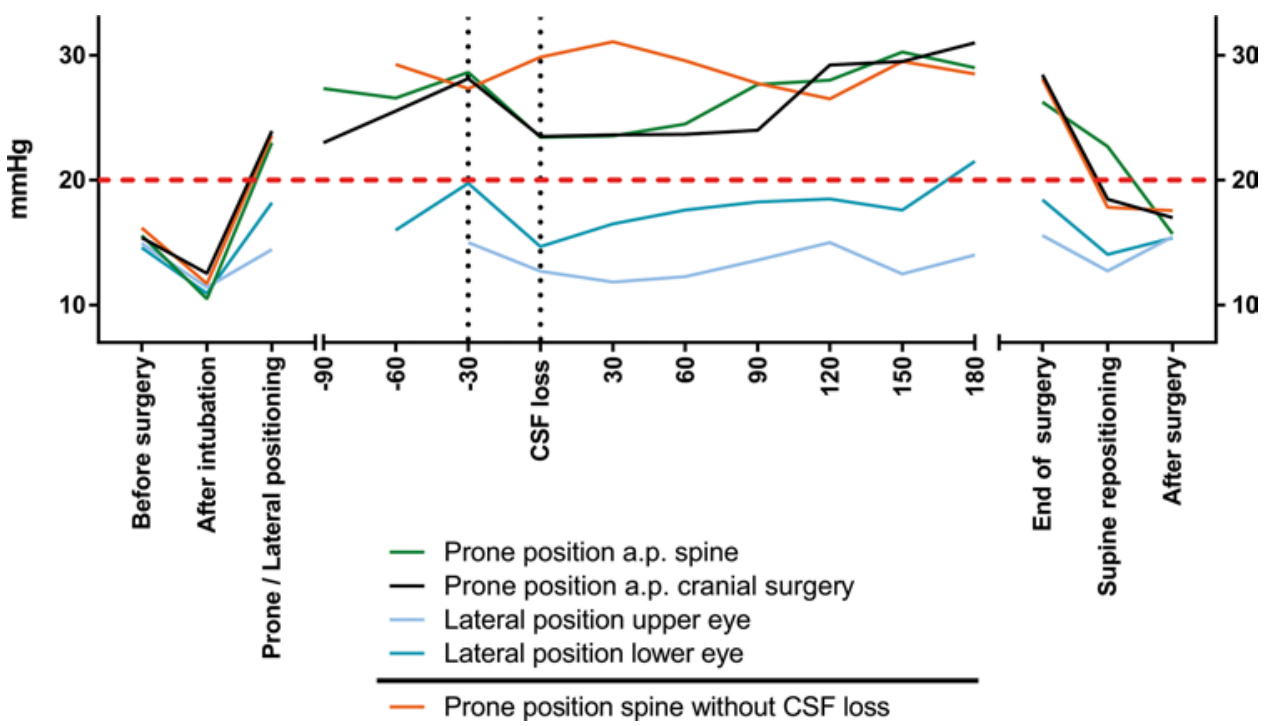

FIG. 3. Time and CSF loss related to the development of IOP. Chart distribution of the IOP courses of groups A, B, and D and for the lower and upper eye in group $C$ throughout the entire surgical procedure. a.p. $=$ anteroposterior.

$\pm 8 \mathrm{~mm} \mathrm{Hg}$ in group $\mathrm{C}$, and $116 \pm 33 / 71 \pm 20 \mathrm{~mm} \mathrm{Hg}$ in group D. Before CSF drainage, the mean BP (BP1) was $107 \pm 13 / 60 \pm 8 \mathrm{~mm} \mathrm{Hg}, 111 \pm 11 / 65 \pm 10 \mathrm{~mm} \mathrm{Hg}$, and 115 $\pm 15 / 60 \pm 10 \mathrm{~mm} \mathrm{Hg}$ for groups A, B, and C, respectively. In group $\mathrm{D}$, the $\mathrm{BP}$ measured at the beginning of surgery (BP1) was $120 \pm 18 / 64 \pm 10 \mathrm{~mm} \mathrm{Hg}$. The BP values were $102 \pm 12 / 58 \pm 8 \mathrm{~mm} \mathrm{Hg}$ (group A), $111 \pm 13 / 62 \pm 8 \mathrm{~mm}$ $\mathrm{Hg}$ (group B), and $113 \pm 13 / 59 \pm 8 \mathrm{~mm} \mathrm{Hg}$ (group C) when the dura was opened (BP2). For group D, the mean BP 60 minutes after the beginning of surgery was $109 \pm 9 / 62 \pm$ $11 \mathrm{~mm} \mathrm{Hg}$ as an alternatively chosen time for comparison (BP2). Overall, there were no statistically significant differences in the mean BP between induction of anesthesia and $\mathrm{BP} 1$ or $\mathrm{BP} 2$ throughout the entire procedure in any group, with $\mathrm{p}=0.1792(\mathrm{BP} 1)$ and $\mathrm{p}=0.3748(\mathrm{BP} 2)$ for group $A, p=0.9199(\mathrm{BP} 1)$ and $\mathrm{p}=0.2926(\mathrm{BP} 2)$ for group $\mathrm{B}, \mathrm{p}=0.4379(\mathrm{BP} 1)$ and $\mathrm{p}=0.5992(\mathrm{BP} 2)$ for group $\mathrm{C}$, and $\mathrm{p}=0.2665(\mathrm{BP} 1)$ and $\mathrm{p}=0.9836(\mathrm{BP} 2)$ for group $\mathrm{D}$. Details are presented in Fig. 4.

\section{Volume of Blood Loss}

There were no significant differences in terms of the amount of blood loss in each group (A: $576.7 \pm 374.6 \mathrm{ml}$; B: $353.1 \pm 363.5 \mathrm{ml} ; \mathrm{C}: 340.6 \pm 257.0 \mathrm{ml}$; and D: $615.0 \pm$ $719.2 \mathrm{ml})$. There was no significant correlation between the amount of blood loss and reduction in IOP measured within the defined time frames of 30 minutes before dura opening to the opening of the dura (Pearson $r=0.049, p$ $=0.749)$ as well as 30 minutes after CSF loss (Pearson $r=$ $0.139, \mathrm{p}=0.350$ ).

\section{Perioperative Volume Management}

No statistically significant differences were seen in the volume of crystalloid fluids applied in any group (A: 2129 $\pm 1388.0 \mathrm{ml}$; B: $2150 \pm 879.4 \mathrm{ml} ; \mathrm{C}: 2053 \pm 1050.0 \mathrm{ml}$; and D: $1922 \pm 1211.0 \mathrm{ml})$. There was no significant correlation between the volume of intravenous crystalloids and the IOP at 30 minutes before CSF loss to dural opening (Pear- son $\mathrm{r}=-0.029, \mathrm{p}=0.849)$ and 30 minutes after opening of the dura mater (Pearson $\mathrm{r}=0.007, \mathrm{p}=0.961$ ).

\section{Ophthalmological Examination}

One patient had known anisocoria but without pupillomotor disturbance. Another patient presented with a pterygium of the cornea preoperatively. The anterior chamber was without pathology in 47 patients, while 15 patients had a cataract. The postoperative ophthalmological examination showed 1 corneal irritation in a single eye requiring no further treatment.

A paired analysis of the visual function did not reveal any significant differences in the preoperative versus postoperative status for the right eye (preoperative mean $0.84 \pm$ 0.22 vs postoperative mean $0.85 \pm 0.21, \mathrm{p}=0.3353$ ) or the left eye (preoperative mean $0.87 \pm 0.20$ vs postoperative mean $0.88 \pm 0.18, \mathrm{p}=0.6279$ ).

\section{Discussion}

To the best of our knowledge, this is the first systematic study demonstrating the relationship between intraoperative opening of the dura mater with loss of CSF and IOP in cranial and spinal neurosurgical procedures. Opening of the dura mater with CSF loss was associated with a statistically significant decrease in IOP, which lasted up to 60 minutes independent from the positioning of the head or the procedure itself.

Intraoperative IOP has only been measured in study designs with an unimpaired dura mater. In 2001 Cheng et al. investigated 20 patients undergoing spine surgery in the prone position and demonstrated that IOP is time dependent. ${ }^{6}$ IOP was measured pre- and perioperatively, prior to anesthesia, in the supine position, and at the beginning and end of prone positioning as well as after supine repositioning. The study protocol on the basis of key time points for IOP measurement is similar to our study, but Cheng and coworkers did not evaluate the impact of CSF loss. 


\section{Blood pressure in Relation to IOP - Group A}

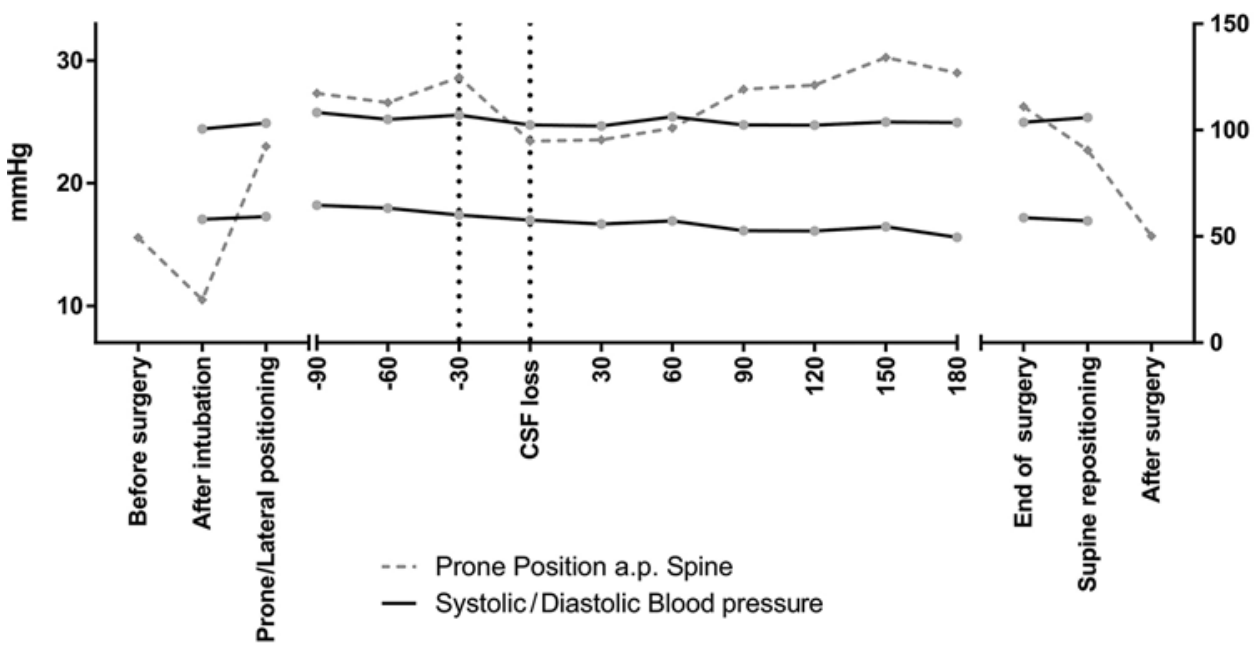

Blood pressure in Relation to IOP - Group B

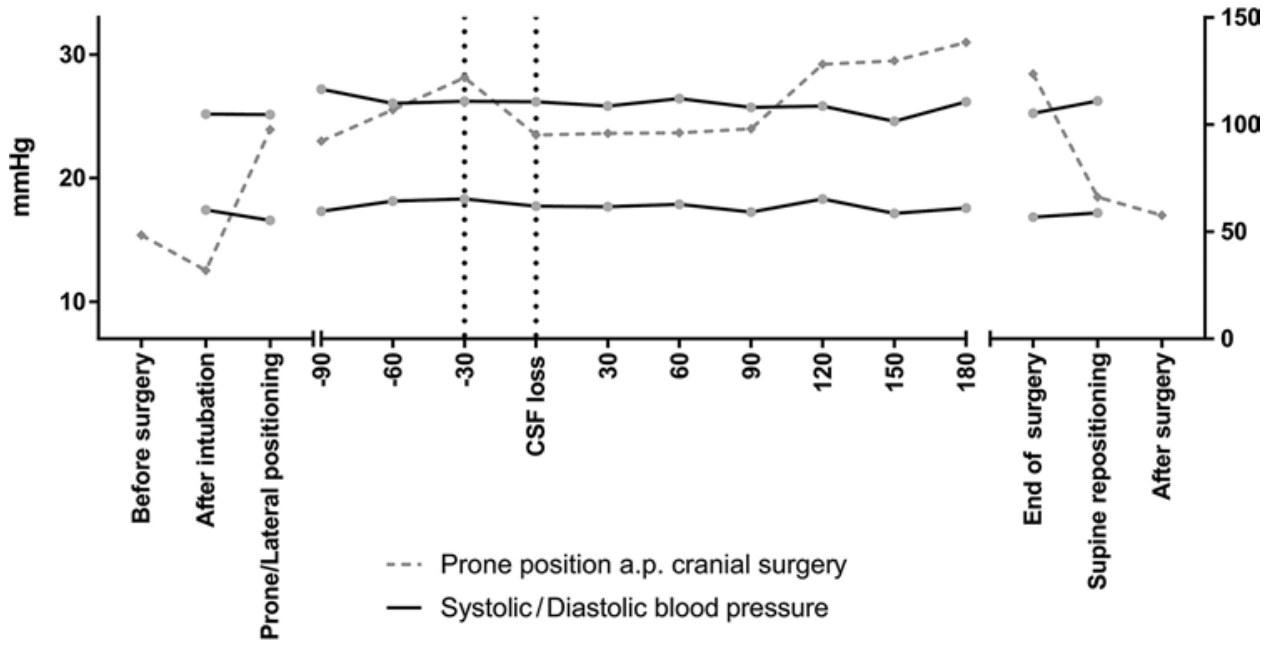

Blood pressure in Relation to IOP - Group C

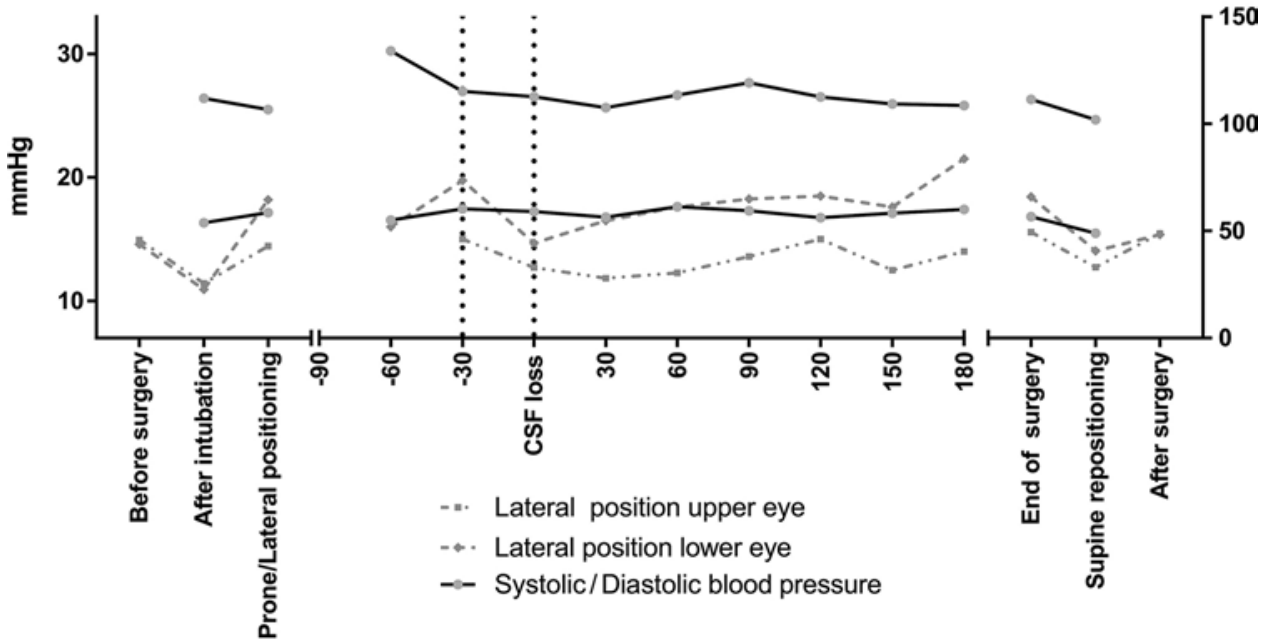

FIG. 4. $B P$ in relation to IOP. The perioperative course of $B P$ in groups $A-C$ in relation to the IOP, demonstrating no significant difference of the BP course. As in group D, no loss of CSF occurred, and IOP remained stable. No further details of BP are presented. 
Other studies have observed an increase in the IOP in the prone position, but have failed to demonstrate a time dependency ${ }^{15}$ That same year, the relationship between a patient's head positioning and IOP was assessed in 10 awake volunteers on 2 different operating tables. ${ }^{31}$ Further studies evaluated the effect of prone versus prone Trendelenburg position, resulting in an additional increase in IOP in the prone Trendelenburg position with time-dependent correlation. ${ }^{43}$ The opposite effect was shown by Carey et al., who randomly assigned 19 patients to different positions with an elevated head of $0^{\circ}, 5^{\circ}$, or $10^{\circ}$. Even a $5^{\circ}$ elevation of the head resulted in a significantly lower IOP than at $0^{\circ}$ positioning. ${ }^{4}$ The impact of head positioning has also been shown in a randomized trial that investigated the effect of a $45^{\circ}$ head rotation in patients undergoing a percutaneous nephrolithotomy, showing a significant difference between the IOP in the upper and lower eyes. ${ }^{8}$

Our data are in line with these previous studies, demonstrating an increased IOP after turning the patient from supine to prone, but we found only a slight and not significant time-dependent additional increase in IOP during surgery in the control group (group D). ${ }^{6,8,15,31,43}$ We also confirmed the results by Deniz et al. with significant differences between the upper and lower eyes in patients with their heads rotated. ${ }^{8}$

This first report about the association of CSF loss and the significant impact on the IOP during surgical procedures adds new insights about the pathogenesis of POVL and might provide a rationale for the development of new prophylactic strategies.

Grant et al. hypothesized that, due to an increased IOP, the intraorbital venous pressure rises and leads to venous congestion. ${ }^{13}$ They found significant differences in the diameter of optic nerve sheaths in 10 awake and healthy volunteers after 5 hours in the prone position, but they could not differentiate between the entire retrobulbar optic nerve sheath complex and the isolated optic nerve sheath. The increase in diameter could be a result of either an increasing CSF pressure or an increasing venous congestion, or the dependent combination of both. Taketani and colleagues had the same finding only 30 minutes after steep Trendelenburg positioning in laparoscopic surgery. ${ }^{40}$ It would have been interesting to measure the diameter of the optic nerve sheath complex in our study design, but, due to the study setting, it was impossible to perform reliable ultrasonographic examinations without disturbing the surgical procedure. Interestingly, 5 hours of $4^{\circ}$ reverse Trendelenburg positioning led to no decrease in IOP or optic nerve sheath diameter but attenuated the choroidal thickness. ${ }^{13}$ The awake volunteers, however, received no crystalloid infusion or anesthesia. It remains unclear if this has an impact on the results, as the American Society of Anesthesiologists Task Force on Perioperative Visual Loss in spinal surgery detected not only head and orbit positioning below the heart and surgery $>6.5$ hours, but also excessive blood loss ( $>44.7 \%$ circulating blood volume) and overhydrating with crystalloids as independent risk factors for POVL.,13 We still state that CSF loss, as in our study groups A-C, which significantly decreased the IOP, would have resulted in a reduction of the optic nerve sheath diameter as both parameters are associated. ${ }^{39}$
Our findings also support the theory that an increasing IOP leads to a reduced optic nerve perfusion pressure, which is calculated as the mean arterial BP minus the IOP, so loss of CSF and a decrease of the IOP result in an increase in the optic nerve perfusion pressure. ${ }^{43}$

As POVL is most commonly described in spine surgery in the prone position, the amount of blood loss during longer surgeries or calculated hypotension might also result in a decreased mean arterial BP and consequently reduced optic nerve perfusion pressure..$^{1,6,18}$ In addition, that blood loss in intracranial or intradural spinal procedures is generally lower than that in complex, often long, spine surgeries might explain the fact that POVL in intracranial, microsurgical procedures is limited to some case reports, as we also identified no case of POVL or visual disturbance in our study. ${ }^{41}$

Other authors, such as Newman, assumed that an increase in venous cerebral pressure leads to an increase in the IOP as well as hypoperfusion of the optic nerve sheath. The author stated that an increased IOP is the consequence and not the cause of this phenomenon. ${ }^{25,30,34}$ Reports from ION after neck dissection surgery with ligation of the jugular veins resulting in an increased intracranial and venous pressure also support this theory. ${ }^{33}$

As a consequence of the recent literature and our findings, it is necessary to develop strategies to avoid an elevation of IOP or lower a raised IOP in patients at risk with preexisting compromised ocular blood flow due to hypertension, diabetes, atherosclerosis, or glaucoma. As described before, positioning of the patient affects the IOP most and can lead to a pathologic increase, levels $>24 \mathrm{~mm}$ $\mathrm{Hg}$, with potentially negative consequences. ${ }^{7}$ There are recommendations on how to avoid IOP elevation if pathology demands surgery in the prone or Trendelenburg position that we support: positioning the head at or above the level of the heart, continuous BP monitoring, blood loss replacement with colloids as well as crystalloids, and periodical hemoglobin or hematocrit assessment. . $^{1,4,9,10,16,27,45}$ The choice of medical anesthetic agents can also influence the IOP. For induction of anesthesia, propofol is known to have the most profound lowering effect of IOP; for maintenance of anesthesia, sevoflurane is equal to propofol..$^{26,29,36}$ For neuromuscular blocking, rocuronium demonstrated a greater reduction in IOP and equally favorable intubating conditions as succinylcholine ${ }^{42}$ Besides optimization of those factors, dorzolamide-timolol ophthalmic solution is an option to reduce IOP. It was applied in 90 patients undergoing surgery in steep Trendelenburg positioning, showing a significantly reduced IOP. ${ }^{27}$

With the results of our study, we have to take into consideration whether prophylactic reduction of CSF by a preoperative lumbar puncture or continuous CSF drainage can reduce IOP during surgery in the prone or Trendelenburg position without planned opening of the dura mater to avoid negative ophthalmological consequences. In patients with idiopathic intracranial hypertension with visual disturbances due to long-term intracranial pressure elevation, medical options to reduce CSF production are available, and serial lumbar punctures are a known therapy that significantly improve vision..$^{5}$ It can be discussed whether CSF drainage is too invasive, but it became routine in the 
past few years during endovascular aortic procedures to reduce and/or avoid the risk of spinal ischemia. Due to the loss of CSF, the intradural spinal pressure is reduced, and an impaired perfusion can be improved. This procedure has verifiable neuroprotective effects. ${ }^{19}$ Further observational studies might discuss the feasibility of an alternative lateral or sitting position instead of prone positioning for surgery to reduce the risk of POVL.

\section{Study Limitations}

The study suffers from some limitations. We did not measure and document the overall angle of patients' positions with the head being elevated or lowered during surgery. We assumed that an IOP decrease, as hypothesized, occurs in every position. Furthermore, there was no measurement of $\mathrm{paCO}_{2}$ in our study as invasive $\mathrm{BP}$ monitoring or measurement of end-tidal $\mathrm{CO}_{2}$ was not administered routinely in all patients. The depth of anesthesia was also not monitored in detail and might have an influence on the IOP values, as induction of anesthesia caused a significant decrease in IOP. Finally, we were not able to measure the perfusion of the retina or the volume of the CSF loss during surgery in the operating room, as our premise was to ensure the patient's safety and avoid any interruption or delay of surgery as well as distraction and disturbance of the neurosurgeon.

\section{Conclusions}

Our study is the first to demonstrate that opening of the dura with loss of CSF during neurosurgical procedures results in a decrease of the IOP. This might explain why POVL predominantly occurs in spine but hardly ever in intradural procedures, offers new insights in the pathophysiology of POVL, and provides the basis for further research and treatment of POVL.

\section{References}

1. American Society of Anesthesiologists Task Force on Perioperative Visual Loss: Practice advisory for perioperative visual loss associated with spine surgery: an updated report by the American Society of Anesthesiologists Task Force on Perioperative Visual Loss. Anesthesiology 116:274-285, 2012

2. Amm M, Hedderich J: [Transpalpebral tonometry with a digital tonometer in healthy eyes and after penetrating keratoplasty.] Ophthalmologe 102:70-76, 2005 (Ger)

3. Berg KT, Harrison AR, Lee MS: Perioperative visual loss in ocular and nonocular surgery. Clin Ophthalmol 4:531-546, 2010

4. Carey TW, Shaw KA, Weber ML, DeVine JG: Effect of the degree of reverse Trendelenburg position on intraocular pressure during prone spine surgery: a randomized controlled trial. Spine J 14:2118-2126, 2014

5. Chan JW: Current concepts and strategies in the diagnosis and management of idiopathic intracranial hypertension in adults. J Neurol 264:1622-1633, 2017

6. Cheng MA, Todorov A, Tempelhoff R, McHugh T, Crowder $\mathrm{CM}$, Lauryssen C: The effect of prone positioning on intraocular pressure in anesthetized patients. Anesthesiology 95:1351-1355, 2001

7. David R, Zangwill L, Briscoe D, Dagan M, Yagev R, Yassur Y: Diurnal intraocular pressure variations: an analysis of 690 diurnal curves. Br J Ophthalmol 76:280-283, 1992
8. Deniz MN, Erakgün A, Sertöz N, Yilmaz SG, Ateş H, Erhan E: The effect of head rotation on intraocular pressure in prone position: a randomized trial. Braz J Anesthesiol 63:209-212, 2013

9. Emery SE, Daffner SD, France JC, Ellison M, Grose BW, Hobbs GR, et al: Effect of head position on intraocular pressure during lumbar spine fusion: a randomized, prospective study. J Bone Joint Surg Am 97:1817-1823, 2015

10. Farag E, Sessler DI, Kovaci B, Wang L, Mascha EJ, Bell G, et al: Effects of crystalloid versus colloid and the $\alpha-2$ agonist brimonidine versus placebo on intraocular pressure during prone spine surgery: a factorial randomized trial. Anesthesiology 116:807-815, 2012

11. Gilbert ME: Postoperative visual loss: a review of the current literature. Neuro-Ophthalmology 32:194-199, 2008

12. Goepfert CE, Ifune C, Tempelhoff R: Ischemic optic neuropathy: are we any further? Curr Opin Anaesthesiol 23:582-587, 2010

13. Grant GP, Szirth BC, Bennett HL, Huang SS, Thaker RS, Heary RF, et al: Effects of prone and reverse Trendelenburg positioning on ocular parameters. Anesthesiology 112:5765,2010

14. Ho VT, Newman NJ, Song S, Ksiazek S, Roth S: Ischemic optic neuropathy following spine surgery. J Neurosurg Anesthesiol 17:38-44, 2005

15. Hunt K, Bajekal R, Calder I, Meacher R, Eliahoo J, Acheson JF: Changes in intraocular pressure in anesthetized prone patients. J Neurosurg Anesthesiol 16:287-290, 2004

16. Kelly DJ, Farrell SM: Physiology and role of intraocular pressure in contemporary anesthesia. Anesth Analg 126:15511562,2018

17. Kendrick H: Post-operative vision loss (POVL) following surgical procedures. J Anesth Clin Res 3:184, 2012

18. Kla KM, Lee LA: Perioperative visual loss. Best Pract Res Clin Anaesthesiol 30:69-77, 2016

19. Kotelis D, Bianchini C, Kovacs B, Müller T, Bischoff M, Böckler D: Early experience with automatic pressure-controlled cerebrospinal fluid drainage during thoracic endovascular aortic repair. J Endovasc Ther 22:368-372, 2015

20. Lee LA: Perioperative visual loss and anesthetic management. Curr Opin Anaesthesiol 26:375-381, 2013

21. Lee LA, Deem S, Glenny RW, Townsend I, Moulding J, An $D$, et al: Effects of anemia and hypotension on porcine optic nerve blood flow and oxygen delivery. Anesthesiology 108:864-872, 2008

22. Lee LA, Newman NJ, Wagner TA, Dettori JR, Dettori NJ: Postoperative ischemic optic neuropathy. Spine (Phila Pa 1976) 35 (9 Suppl):S105-S116, 2010

23. Lee LA, Roth S, Posner KL, Cheney FW, Caplan RA, Newman NJ, et al: The American Society of Anesthesiologists Postoperative Visual Loss Registry: analysis of 93 spine surgery cases with postoperative visual loss. Anesthesiology 105:652-659, 867-868, 2006

24. Lee LA, Roth S, Todd MM: Risk factors associated with ischemic optic neuropathy after spinal fusion surgery. Anesthesiology 116:15-24, 2012

25. Lirng JF, Fuh JL, Wu ZA, Lu SR, Wang SJ: Diameter of the superior ophthalmic vein in relation to intracranial pressure. AJNR Am J Neuroradiol 24:700-703, 2003

26. Mirakhur RK, Shepherd WF, Darrah WC: Propofol or thiopentone: effects on intraocular pressure associated with induction of anaesthesia and tracheal intubation (facilitated with suxamethonium). Br J Anaesth 59:431-436, 1987

27. Molloy BL, Cong X, Watson C: Preventive dorzolamidetimolol for rising intraocular pressure during steep Trendelenburg position surgery. AANA J 84:189-196, 2016

28. Myers MA, Hamilton SR, Bogosian AJ, Smith CH, Wagner TA: Visual loss as a complication of spine surgery. A review of 37 cases. Spine (Phila Pa 1976) 22:1325-1329, 1997 
29. Neel S, Deitch R Jr, Moorthy SS, Dierdorf S, Yee R: Changes in intraocular pressure during low dose intravenous sedation with propofol before cataract surgery. Br J Ophthalmol 79:1093-1097, 1995

30. Newman NJ: Perioperative visual loss after nonocular surgeries. Am J Ophthalmol 145:604-610, 2008

31. Ozcan MS, Praetel C, Bhatti MT, Gravenstein N, Mahla ME, Seubert CN: The effect of body inclination during prone positioning on intraocular pressure in awake volunteers: a comparison of two operating tables. Anesth Analg 99:11521158,2004

32. Patil CG, Lad EM, Lad SP, Ho C, Boakye M: Visual loss after spine surgery: a population-based study. Spine (Phila Pa 1976) 33:1491-1496, 2008

33. Pazos GA, Leonard DW, Blice J, Thompson DH: Blindness after bilateral neck dissection: case report and review. Am J Otolaryngol 20:340-345, 1999

34. Reitsamer HA, Kiel JW, Harrison JM, Ransom NL, McKinnon SJ: Tonopen measurement of intraocular pressure in mice. Exp Eye Res 78:799-804, 2004

35. Rinaldi I, Botton JE, Troland CE: Cortical visual disturbances following ventriculography and/or ventricular decompression. J Neurosurg 19:568-576, 1962

36. Sator-Katzenschlager S, Deusch E, Dolezal S, MichalekSauberer A, Grubmüller R, Heinze G, et al: Sevoflurane and propofol decrease intraocular pressure equally during nonophthalmic surgery and recovery. Br J Anaesth 89:764-766, 2002

37. Shen Y, Drum M, Roth S: The prevalence of perioperative visual loss in the United States: a 10-year study from 1996 to 2005 of spinal, orthopedic, cardiac, and general surgery. Anesth Analg 109:1534-1545, 2009

38. Singh S, Dass R: The central artery of the retina. I. Origin and course. Br J Ophthalmol 44:193-212, 1960

39. Singleton J, Dagan A, Edlow JA, Hoffmann B: Real-time optic nerve sheath diameter reduction measured with bedside ultrasound after therapeutic lumbar puncture in a patient with idiopathic intracranial hypertension. Am J Emerg Med 33:860.e5-860.e7, 2015

40. Taketani Y, Mayama C, Suzuki N, Wada A, Oka T, Inamochi $\mathrm{K}$, et al: Transient but significant visual field defects after robot-assisted laparoscopic radical prostatectomy in deep Trendelenburg position. PLoS One 10:e0123361, 2015

41. Vahedi P, Meshkini A, Mohajernezhadfard Z, Tubbs RS: Post-craniotomy blindness in the supine position: Unlikely or ignored? Asian J Neurosurg 8:36-41, 2013

42. Vinik HR: Intraocular pressure changes during rapid sequence induction and intubation: a comparison of ro- curonium, atracurium, and succinylcholine. J Clin Anesth 11:95-100, 1999

43. Walick KS, Kragh JE Jr, Ward JA, Crawford JJ: Changes in intraocular pressure due to surgical positioning: studying potential risk for postoperative vision loss. Spine (Phila Pa 1976) 32:2591-2595, 2007

44. Williams EL, Hart WM Jr, Tempelhoff R: Postoperative ischemic optic neuropathy. Anesth Analg 80:1018-1029, 1995

45. Yamada MH, Takazawa T, Iriuchijima N, Horiuchi T, Saito S Changes in intraocular pressure during surgery in the lateral decubitus position under sevoflurane and propofol anesthesia J Clin Monit Comput 30:869-874, 2016

\section{Disclosures}

The authors report no conflict of interest concerning the materials or methods used in this study or the findings specified in this paper.

\section{Author Contributions}

Conception and design: Czorlich, Kluge, Skevas, Eicker. Acquisition of data: Czorlich, Krätzig, Kluge, Skevas, Knospe, Eicker. Analysis and interpretation of data: Czorlich, Eicker. Drafting the article: Czorlich, Krätzig, Skevas, Mende, Eicker. Critically revising the article: Czorlich, Krätzig, Kluge, Skevas, Knospe, Spitzer, Dreimann, Westphal, Eicker. Reviewed submitted version of manuscript: Czorlich, Krätzig, Kluge, Westphal, Eicker. Approved the final version of the manuscript on behalf of all authors: Czorlich. Statistical analysis: Mende. Administrative/technical/material support: Czorlich. Study supervision: Czorlich, Eicker.

\section{Supplemental Information}

\section{Previous Presentations}

Portions of this work were presented at the Spinal Section Meeting of the German Society of Neurosurgery (DGNC), Berlin, Germany, October 2-3, 2015; the 41st Section Meeting for Intracranial Pressure, Cerebral Perfusion and Hydrocephalus of the DGNC Giessen, Germany, November 6-7, 2015; the 10th Annual Meeting of the German Spine Society, Frankfurt/Main, Germany, December 10-12, 2015; and the 67th Annual Meeting of the DGNC held in Frankfurt/Main, Germany, June 12-15, 2016.

\section{Correspondence}

Patrick Czorlich: University Medical Center Hamburg-Eppendorf, Hamburg, Germany.p.czorlich@uke.de. 